\title{
Generalizations of the Sugawara Model*
}

\author{
S. Deser and J. Rawls $\dagger$ \\ Brandeis University, Waltham, Massachusetts 02154
}

(Received 7 May 1969)

\begin{abstract}
Two generalizations of Sugawara models are discussed. In the first, new interactions are introduced by altering the current commutators but retaining the "free" form of $T_{\mu \nu}$, as exemplified by a simple effective $\pi^{0}-2 \gamma$ interaction, which is compared with the standard triangle-diagram results. The second permits nonquadratic $T_{\mu \nu}$, which retain the "algebra" part of the current commutators; these models avoid the DashenFrishman parity degeneracy.
\end{abstract}

\section{INTRODUCTION}

$T$ HE dynamical theory of currents, as originally proposed by Sugawara ${ }^{1}$ and Sommerfield, ${ }^{2}$ is specified entirely by a stress tensor $T_{\mu \nu}$ and equal-time commutation relations among the "currents" from which $T_{\mu \nu}$ is formed. Considerable work $^{3-6}$ has been done on the formal structure of particular models in which $T_{\mu \nu}$ is quadratic in currents whose commutators are those of the algebra of fields (we refer to this as the Sugawara theory). In this paper, we discuss two generalizations of the theory. In Sec. II, we introduce interactions within the framework of an apparently free, quadratic $T_{\mu \nu}$ by altering the equal-time current commutation relations. In particular, we display a model whose $T_{\mu \nu}$ is the sum of Sugawara's and Maxwell's, such that $\partial^{\mu} A_{\mu}=(\alpha / 2 \pi) \bar{F}^{\mu \nu} F_{\mu \nu}$, where $A_{\mu}$ is the neutral axialvector current. We shall compare this simple model of an effective $\pi^{0}-2 \gamma$ coupling with recent detailed analy$\operatorname{ses}^{7-9}$ of the process in terms of intermediate fermion loops, ${ }^{10}$ since the latter also effectively alter the commutation relations of the original field theory.

In Sec. III, we deal with $T^{\mu \nu}$ that are not quadratic in the currents, but retain $S U_{n} \times S U_{n}$ invariance and have the Sugawara model as a limiting case. The techniques of both the Lagrangian formulation ${ }^{4,11}$ and the Yang-Mills limiting process ${ }^{3}$ apply as in the original model. The current commutators differ from those of the algebra of fields in their "nonalgebra" parts, namely, in the space-time Schwinger terms and spacespace commutators. Such models will be shown to avoid the difficulty recently pointed out by Dashen and

* Research supported in part by the U. S. Air Force, OAR, under Grant No. OSR 368-67.

† National Science Foundation Predoctoral Fellow.

1 H. Sugawara, Phys. Rev. 170, 1659 (1968).

2 C. Sommerfield, Phys. Rev. 176, 2019 (1968).

${ }^{3}$ K. Bardakci, Y. Frishman, and M. B. Halpern, Phys. Rev. 170, 1353 (1968)

${ }^{4} \mathrm{~K}$. Bardakci and M. B. Halpern, Phys. Rev. 172, 1542 (1968).

${ }^{5}$ S. Coleman, D. Gross, and R. Jackiw, Phys. Rev. 180, 1359 (1969).

${ }^{6}$ D. J. Gross and M. B. Halpern, Phys. Rev. 179, 1436 (1969).

${ }^{7}$ S. L. Adler, Phys. Rev. 177, 2426 (1969).

${ }^{8} \mathrm{R}$. Jackiw and K. Johnson, Phys. Rev. 182, 1459 (1969).

${ }^{9} \mathrm{~S}$. L. Adler and D. G. Boulware, Phys. Rev. 184, 1740 (1969).

${ }^{10}$ Such problems were first dealt with by J. Steinberger, Phys.

Rev. 76, 1180 (1949); and J. Schwinger, ibid. 82, 664 (1951).

11 S. Deser, preceding paper, Phys. Rev. 187, 1931 (1969).

187
Frishman, ${ }^{12}$ from which the Sugawara theory suffers: There $S U_{n} \times S U_{n}$ symmetry entails a $\mathcal{P}_{+} \times \mathcal{P}_{-}$symmetry under the product of two independent Poincaré groups and, correspondingly, a parity doubling of particle states. In the generalized models, we will see that the internal symmetry no longer implies Poincaré degeneracy.

\section{INTERACTIONS VIA CURRENT COMMUTATORS}

Consider the Lagrangian ${ }^{13}$

$$
\begin{gathered}
\mathcal{L}=-\frac{1}{2} \partial_{\mu} \phi \partial^{\mu} \phi-\frac{1}{4} F^{\mu \nu} F_{\mu \nu}-(\alpha / 2 \pi) \phi \bar{F}^{\mu \nu} F_{\mu \nu}, \\
F_{\mu \nu} \equiv \partial_{\mu} Q_{\nu}-\partial_{\nu} Q_{\mu}, \\
\bar{F}^{\mu \nu} \equiv \frac{1}{2} \epsilon^{\mu \nu \sigma \rho} F_{\sigma \rho}=\epsilon^{\mu \nu \sigma \rho} \partial_{\sigma} Q_{\rho},
\end{gathered}
$$

where $\phi$ is a neutral pseudoscalar field, and $Q_{\mu}$ is the vector potential. ${ }^{14}$ Our metric $\eta_{\mu \nu}$ is $(-+++)$, and we adopt the convention $\epsilon^{0123}=1$. The coupling constant is of course an arbitrary parameter in our approach (which is not based on a fundamental minimal electromagnetic coupling) and has been written as $\alpha / 2 \pi$ to yield the usual perturbation-theory result. The nonminimal term $\phi \bar{F} F$ in Eq. (1) is metric-independent and hence does not contribute to $T_{\mu \nu}$ : When the action is written in generally covariant form, the stress tensor is simply the response to a change in the metric

$$
T^{\mu \nu}=2 \delta I / \delta g_{\mu \nu} .
$$

Since $\phi \bar{F}^{\mu \nu} F_{\mu \nu}=\frac{1}{2} \phi \epsilon^{\mu \nu \sigma \rho} F_{\sigma \rho} F_{\mu \nu}$ is already a scalar density ( $\epsilon^{\mu \nu \sigma \rho}$ is a metric-independent tensor density), it will not contribute, and one obtains the form

$$
T_{\mu \nu}=\partial_{\mu} \phi \partial_{\nu} \phi-\frac{1}{2} \eta_{\mu \nu} \partial_{\sigma} \phi \partial^{\sigma} \phi+F_{\mu \sigma} F_{\nu}{ }^{\sigma}-\frac{1}{4} \eta_{\mu \nu} F^{\sigma \rho} F_{\sigma \rho},
$$

which does not involve the interaction. The field equations are

$$
\square \phi=(\alpha / 2 \pi) \bar{F}^{\mu \nu} F_{\mu \nu}, \quad \partial_{\nu} F^{\mu \nu}=-(2 \alpha / \pi) \partial_{\nu} \phi \bar{F}^{\mu \nu} .
$$

${ }^{12}$ R. Dashen and Y. Frishman, Phys. Rev. Letters 22, 572 (1969).

${ }^{13}$ An alternative starting point is the first-order Lagrangian formulation for the $A_{\mu}: I=\int\left(-\frac{1}{2} A^{\mu} A_{\mu}+A^{\mu} \partial_{\mu} \phi\right)-\frac{1}{4} \int F^{\mu \nu} F_{\mu \nu}$ $+(\alpha / 2 \pi) \int_{\phi} \bar{F}^{\mu \nu} F_{\mu \nu}$, where the current form is built in.

${ }_{14}$ Throughout, we ignore ordering problems at a point; they are no different from those encountered in the Sugawara model and can be similarly dealt with. 
If we define the axial-vector current by

$$
A_{\mu}=\partial_{\mu} \phi,
$$

the field equations become

$$
\begin{gathered}
\partial_{\mu} A^{\mu}=(\alpha / 2 \pi) \bar{F}^{\mu \nu} F_{\mu \nu}, \quad \partial_{\mu} A_{\nu}-\partial_{\nu} A_{\mu}=0, \\
\partial_{\nu} F^{\mu \nu}=-(2 \alpha / \pi) A_{\nu} \bar{F}^{\mu \nu} \equiv e j^{\mu},
\end{gathered}
$$

and $T_{\mu \nu}$ takes on the Sugawara form of the Abelian theory:

$$
\begin{aligned}
T_{\mu \nu} & =T_{\mu \nu}(A)+T_{\mu \nu}(F) \\
& =A_{\mu} A_{\nu}-\frac{1}{2} \eta_{\mu \nu} A_{\sigma} A^{\sigma}+F_{\mu \sigma} F_{\nu}{ }^{\sigma}-\frac{1}{4} \eta_{\mu \nu} F^{\sigma \rho} F_{\sigma \rho} .
\end{aligned}
$$

Since the field equations are simply the Heisenberg equations

$$
\partial_{\mu} \mathcal{O}(x)=i \int d^{3} y\left[\mathcal{O}(x), T_{\mu}^{0}(y)\right]_{x_{0}=y_{0}},
$$

we have, in the language of the dynamical theory of currents, introduced the interaction via the commutation relations. Indeed, we may now drop the original Lagrangian form and define the theory by the stress tensor (7), along with a set of equal-time commutators among the currents $A_{\mu}, F_{\mu \nu}$, the latter being equivalent to the canonical commutation relations of the original field-theoretical model. We then obtain certain anomalous commutators, due to the gradient coupling in the interaction, which implies that the canonical Maxwell momentum is no longer $F_{0 i}$ but $\pi_{i}=F_{0 i}+(2 \alpha / \pi) \phi \bar{F}_{0 i}$. The commutators then read

$$
\begin{gathered}
{\left[A_{0}(x), A_{0}(y)\right]=\left[A_{i}(x), A_{j}(y)\right]=0,} \\
{\left[A_{0}(x), A_{i}(y)\right]=i \partial_{x_{i}} \delta(\mathbf{x}-\mathbf{y}) ;} \\
{\left[A_{i}(x), F_{\mu \nu}(y)\right]=\left[A_{0}(x), F_{i j}(y)\right]=0,} \\
{\left[F_{i j}(x), F_{k l}(y)\right]=0,} \\
{\left[F_{0 i}(x), F_{j k}(y)\right]=i \delta_{i k} \partial_{x_{j}} \delta(\mathbf{x}-\mathbf{y})-i \delta_{i j} \partial_{x_{k}} \delta(\mathbf{x}-\mathbf{y}) ;} \\
{\left[A_{0}(x), F_{0 i}(y)\right]=(2 i \alpha / \pi) \bar{F}_{0 i}(x) \delta(\mathbf{x}-\mathbf{y}),} \\
{\left[F_{0 i}(x), F_{0 j}(y)\right]=-(2 i \alpha / \pi) \epsilon_{0 i j k} A^{k}(x) \delta(\mathbf{x}-\mathbf{y}) .}
\end{gathered}
$$

Only Eq. (9c) differs from the corresponding commutators of the sum of two free Sugawara models (we refer to free electrodynamics as a Sugawara theory). In fact, the above is a special case of Sommerfield's ${ }^{2}$ general analysis of models of Abelian currents with quadratic $T_{\mu \nu}$. Thus we have here a simple model for an effective massless pion- $2 \gamma$ interaction, introduced through the anomalous commutators.

Let us note an interesting feature of the field equations (6). The now nonconserved axial current may be replaced by another, conserved, quantity: $\bar{F}^{\mu \nu} F_{\mu \nu}$ $=2 \partial_{\mu}\left(\bar{F}^{\mu \nu} Q_{\nu}\right)$ implies that the $\partial_{\mu} A^{\mu}$ equation may be rewritten as

$$
\partial_{\mu}\left[A^{\mu}-(\alpha / \pi) \bar{F}^{\mu \nu} Q_{\nu}\right]=0,
$$

with the conserved axial charge

$$
\bar{Q}^{5}=\int\left[A_{0}(x)-(\alpha / \pi) \bar{F}_{0 i}(x) \mathscr{Q}_{i}(x)\right] d^{3} \mathbf{x}
$$

As noted by Adler, ${ }^{7} \bar{Q}^{5}$ is gauge-invariant, although the integrand is not. This charge ${ }^{15}$ is, in our model, rather trivial in that $\bar{Q}^{5}$ commutes both with the Maxwell variables $F^{\mu \nu}$ and the axial current $A_{\mu}$. It fails to commute only with $\phi$;

$$
i\left[\bar{Q}^{5}, \phi\right]=1 \text {. }
$$

This relation shows that the origin of $\bar{Q}^{5}$ is in the invariance of the massless $\phi$ field under translations, $\phi \rightarrow \phi+$ const, an invariance preserved by the gradient coupling, since

$$
\int \phi \bar{F}^{\mu \nu} F_{\mu \nu} \rightarrow(\text { const }) \int \partial_{\mu}\left(\bar{F}^{\mu \nu} Q_{\nu}\right)=0
$$

In the detailed treatment of the $\pi^{0}-2 \gamma$ interaction in terms of intermediate fermion triangles, ${ }^{7,9}$ the same quantity is also conserved and commutes with $F_{\mu \nu}$, but there generates a chiral transformation on the fermion fields (which leaves the fermion axial-vector current invariant). Note also that there is a nontrivial conserved electric current $j^{\mu}=-\left(e / 2 \pi^{2}\right) A_{\nu} \bar{F}^{\mu \nu}$ even though $A_{\mu}$ is neutral.

In view of the simplicity of this $\pi^{0}$ decay model, which has replaced the intermediate fermion loop by an effective contact interaction [so that the $\partial_{\mu} A^{\mu}=(\alpha / 2 \pi) \bar{F}^{\mu \nu} F_{\mu \nu}$ condition is automatically valid to all orders], it is interesting that the two models have a number of detailed features in common. In particular, Adler and Boulware $^{9}$ have emphasized that certain anomalous commutators are characteristic of the singular nature of the process. We now show that similar commutators appear in the present model, which provides a simple canonical analog to theirs. In order to avoid a special gauge, we transcribe their relations in terms of $F_{\mu \nu}$ (or, equivalently, in terms of $E_{i} \equiv F^{0 i}$ and $B_{i} \equiv \bar{F}^{0 i}$ ) rather than $Q_{\mu}$ and $\partial_{0} Q_{\mu}$. There are two sets of anomalous relations, one of which is exact to all orders ${ }^{15 a}$ of $\alpha$,

$$
\begin{aligned}
& {\left[E_{i}(x), A_{0}(y)\right]=-(2 i \alpha / \pi) B_{i}(y) \delta(\mathbf{x}-\mathbf{y}),} \\
& {\left[j_{0}(x), A_{0}(y)\right]=-\left(i e / 2 \pi^{2}\right) \mathbf{B}(y) \cdot \boldsymbol{\nabla}_{y} \delta(\mathbf{x}-\mathbf{y}) ;}
\end{aligned}
$$

the others are calculated only to lowest order and, in addition, are determined only up to an arbitrary

${ }^{15}$ Strictly speaking, $\bar{Q}^{5}$ is well-defined for massless mesons only in a finite volume; see R. F. Streater, High Energy Physics and Elementary Particles (International Atomic Energy Agency, Vienna, 1965).

15a The treatment of higher orders involves certain formal properties of three-point functions; this may be a delicate point. See R. Jackiw, CERN Report (unpublished) 
pseudotensor operator $S^{i j}$

$$
\begin{array}{r}
{\left[E_{i}(x), A_{j}(y)\right]=(i \alpha / \pi) \epsilon^{i j k} E_{k}(y) \delta(\mathbf{x}-\mathbf{y})} \\
-i e S^{i j}(y) \delta(\mathbf{x}-\mathbf{y}), \\
{\left[j_{0}(x), A_{i}(y)\right]=-\left(i e / 4 \pi^{2}\right) \epsilon^{i j k} E_{j}(y) \partial_{x k} \delta(\mathbf{x}-\mathbf{y})} \\
+i S^{j i}(y) \partial_{x j} \delta(\mathbf{x}-\mathbf{y}),
\end{array}
$$

and

$$
\begin{array}{r}
{\left[j_{i}(x), A_{0}(y)\right]=\left(i e / 4 \pi^{2}\right) \epsilon^{i i k} E_{j}(x) \partial_{y k} \delta(\mathbf{x}-\mathbf{y})} \\
-i S^{i j}(x) \partial_{y j} \delta(\mathbf{x}-\mathbf{y}) .
\end{array}
$$

The first set, Eq. (14a), is identical with Eqs. (9) in our model. In Eq. (14b), the choice $S^{i j}=\left(e / 4 \pi^{2}\right) \epsilon^{i j k} E_{k}$ results in agreement with our commutators: $\left[E_{i}, A_{j}\right]=0$ $=\left[j_{0}, A_{i}\right]$. The only difference occurs in Eq. (14c), which reads (with the above choice of $S^{i j}$ )

$$
\left[j_{i}(x), A_{0}(y)\right]=-\left(i e / 2 \pi^{2}\right)\left[\mathbf{E}(x) \times \nabla_{x}\right]_{i} \delta(\mathbf{x}-\mathbf{y}),
$$

whereas we have an additional term:

$$
\begin{aligned}
{\left[j_{i}(x), A_{0}(y)\right]=} & -\left(i e / 2 \pi^{2}\right)\left[\mathbf{E}(x) \times \boldsymbol{\nabla}_{x}\right]_{i} \delta(\mathbf{x}-\mathbf{y}) \\
& -\left(i e \alpha / \pi^{3}\right)[\mathbf{A}(x) \times \mathbf{B}(x)]_{i} \delta(\mathbf{x}-\mathbf{y}) .
\end{aligned}
$$

The latter may be traced to our explicitly nonminimal coupling, which replaces (-E) by the canonical variable $\boldsymbol{\pi}=-\mathbf{E}-(2 \alpha / \boldsymbol{\pi}) \boldsymbol{\phi} \mathbf{B}$. Indeed, if we formally replace $(-\mathbf{E})$ by $\boldsymbol{\pi}$ in $j_{i}$, we would get, on the right, $(\boldsymbol{\pi} \times \boldsymbol{\nabla}) \boldsymbol{\delta}$. [In any case, since (15a) was calculated in perturbation theory, it is only meaningful to compare the leading terms in Eq. (15), and these agree.] It would seem, then, that at least some of the anomalous features stem simply from the existence of an effective momentum-dependent $\bar{F} F$ coupling irrespective of the underlying mechanism: This is the case for the properties of $\bar{Q}^{5}$ and the commutators of Eqs. (14a), while model dependence enters through the freedom of $S^{i j}$ in (14b) and (14c). We have not investigated whether there are anomalies ${ }^{9}$ in the triple time-ordered products $T\left(A_{\mu} j_{\nu} j_{\alpha}\right)$ in the present models. Note that $j_{i}$ depends explicitly on $\mathbf{E}$, so that the usual assumption ${ }^{16}$ that $\left[j_{i}, a_{j}\right]$ vanishes no longer holds here. Differences then arise between the two models in some commutators involving $j_{\mu}$. For example, the Jacobi identity for $\mathbf{E}, j_{0}$, and $A_{0}$ is satisfied in our theory but not in theirs, even though their calculation uses only model- (i.e., $S^{i j_{-}}$) independent commutators, which are good to all orders. ${ }^{15 \mathrm{a}} \mathrm{We}$ also find that $\left[j_{i}, j_{\mu}\right]$ possess operator Schwinger terms and that all the Jacobi identities we have calculated, including $\left(F_{\mu \nu}, j_{\sigma}, A_{\rho}\right)$, $\left(j_{\mu}, j_{\nu}, A_{\sigma}\right)$, and $\left(j_{\mu}, A_{\nu}, A_{\sigma}\right)$, are satisfied. It is perhaps not surprising that the identities hold here, since we are not considering corrections, but only "Born" diagrams in our model, by calculating all commutators directly from the canonical commutation relations.

${ }^{16}$ D. G. Boulware and L. S. Brown, Phys. Rev. 156, 1724 (1967); D. G. Boulware and S. Deser, ibid. 151, 1278 (1966).
The above model is a realization of a formal limit of the Lagrangian

$$
\begin{gathered}
\mathscr{L}=-\frac{1}{4} \phi^{5}{ }_{\mu \nu} \phi^{5 \mu \nu}-\frac{1}{2} m_{0}{ }^{2} \phi^{5}{ }_{\mu} \phi^{5 \mu}-\frac{1}{4} F^{\mu \nu} F_{\mu \nu} \\
+(\alpha / 2 \pi) \phi^{5}{ }_{\mu} \bar{F}^{\mu \nu} Q_{\nu}, \\
\phi^{5}{ }_{\mu \nu} \equiv \partial_{\mu} \phi^{5}{ }_{\nu}-\partial_{\nu} \phi^{5}{ }_{\mu},
\end{gathered}
$$

where $\phi^{5}{ }_{\mu}$ is a neutral axial-vector field. Defining an axial vector current by

$$
A_{\mu}=m_{0} \phi^{5}{ }_{\mu}
$$

the limit $m_{0} \rightarrow 0$ yields Eqs. (6), (7), and (9). Incidentally, the interaction $\phi^{5}{ }_{\mu} \bar{F}^{\mu \nu} Q_{\nu}$ in (16) is gaugeinvariant, since $\phi_{\mu}^{5} \bar{F}^{\mu \nu}$ is conserved in the limit by virtue of the field equation $\partial_{\mu} \phi_{\nu}^{5}-\partial_{\nu} \phi^{5}{ }_{\mu}=0$. That Eq. (1) is a realization of this theory can be seen by solving the field equations

$$
\partial_{\mu} A_{\nu}-\partial_{\nu} A_{\mu}=0
$$

with $A_{\mu}=\partial_{\mu} \phi$. A straightforward generalization to $S U_{2} \times S U_{2}$ would then seem to be furnished by a limit of the massive $S U_{2} \times S U_{2}$ Yang-Mills theory, together with an interaction term $A_{3 \mu} \bar{F}^{\mu \nu} Q_{\nu}$. However, one of the field equations in this case is (omitting the normal minimal electromagnetic interaction)

$$
\partial_{\nu} F^{\mu \nu}=-(2 \alpha / \pi) A_{3 \nu} \bar{F}^{\mu \nu},
$$

whose divergence is seen to be consistent only when

$$
\partial_{\mu} A_{3 \nu}-\partial_{\nu} A_{3 \mu}=0 \text {, }
$$

i.e., only in the Abelian case. Alternatively, the coupling $A_{3 \mu} \bar{F}^{\mu \nu} \dot{Q}_{\nu}$ is no longer gauge-invariant in the nonAbelian case. Thus a more complicated coupling would seem to be required; one example is given in the Appendix.

\section{NONQUADRATIC $T_{\mu \nu}$}

As Dashen and Frishman ${ }^{12}$ have shown, the energymomentum tensor in Sugawara's theory, ${ }^{17}$ denoted by $T^{S}{ }_{\mu \nu}$,

$$
\begin{array}{r}
T_{\mu \nu}^{S}=m_{0}{ }^{2}\left(\phi_{a \mu} \phi_{a \nu}+\phi_{a \mu}^{5} \phi_{a \nu}^{5}\right) \\
-\frac{1}{2} \eta_{\mu \nu}\left(\phi_{a \lambda} \phi_{a}{ }^{\lambda}+\phi^{5}{ }_{a \lambda} \phi_{a}^{5}{ }_{a}^{\lambda}\right),
\end{array}
$$

where the $\phi_{a \mu}$ and $\phi_{a \mu}^{5}$ are $n^{2}-1$ vector and axial-vector fields, respectively, can be written as the sum of two separately conserved, commuting tensors that are interchanged by parity,

$T^{S_{\mu \nu}}=T^{+}{ }_{\mu \nu}+T^{-}{ }_{\mu \nu}, \partial^{\mu} T^{ \pm}{ }_{\mu \nu}=0, P T^{ \pm}{ }_{\mu \nu} P^{-1}=T^{\mp}{ }_{\mu \nu}$.

The resulting symmetry group $P_{+} \times P_{-}$implies that the states of the theory come in pairs, degenerate in mass but with opposite parity and charge-conjugation properties. It has been suggested that there exists no theory of currents that is $S U_{3} \times S U_{3}$-invariant but not

\footnotetext{
${ }^{17}$ Equation (21) is written here in terms of fields, which (in Sugawara's theory) are proportional to the currents $V$ and $A$ : $\phi_{a \mu}=g_{0} m_{0}^{-2} V_{a \mu}$ and $\phi_{a \mu}^{5}=g_{0} m_{0}{ }^{-2} A_{a \mu}$.
} 
$P_{+} \times P_{-}$-invariant. We shall see that this problem disappears in nonquadratic $T_{\mu \nu}$ models.

It is convenient to take a limiting form of a massive Yang-Mills type of Lagrangian. Our first model, with a quartic $T_{\mu \nu}$, is a limit of the Lagrangian

$$
\mathfrak{L}=-\frac{1}{4}\left(\phi_{a \mu \nu} \phi_{a}{ }^{\mu \nu}+\phi^{5}{ }_{a \mu \nu} \phi_{a^{5}}{ }^{\mu \nu}\right)-\frac{1}{2} m_{0}{ }^{2} I^{2}+\gamma m_{0}{ }^{4} I^{4},
$$

where

$$
\begin{aligned}
\phi_{a \mu \nu} & \equiv \partial_{\mu} \phi_{a \nu}-\partial_{\nu} \phi_{a \mu}+g_{0} c_{a b c}\left(\phi_{b \mu} \phi_{c \nu}+\phi_{b \mu}^{5} \phi_{c \nu}^{5}\right), \\
\phi_{a \mu \nu}^{5} & \equiv \partial_{\mu} \phi_{a \nu}^{5}-\partial_{\nu} \phi_{a \mu}^{5}+g_{0} c_{a b c}\left(\phi_{b \mu} \phi^{5}{ }_{c \nu}+\phi^{5}{ }_{b \mu} \phi_{c \nu}\right), \\
I^{2} & \equiv \phi_{a \mu} \phi_{a}{ }^{\mu}+\phi^{5}{ }_{a \mu} \phi^{5}{ }_{a}{ }^{\mu} .
\end{aligned}
$$

The canonical variables are the same as in the Lagrangian of the algebra of fields, ${ }^{18}$ which is the case $\gamma=0$. The field equations are

$$
\begin{array}{r}
\partial_{\nu} \phi_{a}{ }^{\mu \nu}=g_{0} c_{a b c}\left(\phi_{b}{ }^{\mu \nu} \phi_{c \nu}+\phi_{b^{5}}{ }^{\mu \nu} \phi^{5}{ }_{c \nu}\right) \\
-m_{0}{ }^{2} \phi_{a}{ }^{\mu}+4 \gamma m_{0}{ }^{4} \phi_{a}{ }^{\mu} I^{2}, \\
\partial_{\nu} \phi^{5}{ }_{a}^{\mu \nu}=g_{0} c_{a b c}\left(\phi^{5} b^{\mu \nu} \phi_{c \nu}+\phi_{b}{ }^{\mu \nu} \phi^{5}{ }_{c \nu}\right) \\
-m_{0}{ }^{2} \phi^{5} a^{\mu}+4 \gamma m_{0}{ }^{4} \phi^{5} a^{\mu} I^{2} .
\end{array}
$$

$\mathscr{L}$ is invariant under the infinitesimal transformations

and under

$$
\begin{gathered}
\phi_{a}{ }^{\mu} \rightarrow \phi_{a}{ }^{\mu}+i c_{a b c} \lambda_{b} \phi_{c}{ }^{\mu}, \\
\phi_{a^{5}}{ }^{\mu} \rightarrow \phi^{5}{ }{ }^{\mu}+i c_{a b c} \lambda_{b} \phi^{5}{ }_{c}{ }^{\mu},
\end{gathered}
$$

$$
\begin{aligned}
\phi_{a}{ }^{\mu} & \rightarrow \phi_{a}{ }^{\mu}+i c_{a b c} \omega_{b} \phi^{5}{ }_{c}^{\mu}, \\
\phi_{a}{ }^{5 \mu} & \rightarrow \phi_{a}{ }^{5 \mu}+i c_{a b c} \omega_{b} \phi_{c}{ }^{\mu} .
\end{aligned}
$$

The conserved currents corresponding to these transformations are, using (25),

$$
\begin{aligned}
J_{a}{ }^{\mu} & =g_{0}{ }^{-1}\left[\partial_{\nu} \phi_{a}{ }^{\mu \nu}+m_{0}{ }^{2} \phi_{a}{ }^{\mu}-4 \gamma m_{0}{ }^{4} \phi_{a}{ }^{\mu} I^{2}\right] \\
J^{5}{ }_{a}{ }^{\mu} & =g_{0}{ }^{-1}\left[\partial_{\nu} \phi^{5}{ }^{\mu \nu}+m_{0}{ }^{2} \phi^{5}{ }^{\mu}{ }^{\mu}-4 \gamma m_{0}{ }^{4} \phi^{5}{ }_{a}{ }^{\mu} I^{2}\right] .
\end{aligned}
$$

Since $\partial_{\nu} \phi^{\mu \nu}$ are $\partial_{\nu} \phi^{5 \mu \nu}$ are divergenceless, one can also form the conserved "currents"

$$
\begin{gathered}
V_{a}{ }^{\mu}=J_{a}{ }^{\mu}-g_{0}{ }^{-1} \partial_{\nu} \phi_{a}{ }^{\mu \nu}=g_{0}{ }^{-1} m_{0}{ }^{2} \phi_{a}{ }^{\mu} \\
\quad-4 \gamma m_{0}{ }^{4} g_{0}{ }^{-1} \phi_{a}{ }^{\mu} I^{2}, \\
A_{a^{\mu}}{ }^{\mu} J^{5}{ }_{a}{ }^{\mu}-g_{0}{ }^{-1} \partial_{\nu} \phi^{5} a^{\mu \nu}=g_{0}{ }^{-1} m_{0}{ }^{2} \phi^{5}{ }^{\mu}{ }^{\mu} \\
-4 \gamma m_{0}{ }^{4} g_{0}{ }^{-1} \phi^{5}{ }_{a}{ }^{\mu} I^{2} .
\end{gathered}
$$

We define the vector and axial-vector currents to be $V$ and $A$, noting that when $\gamma=0$, this reduces to the procedure used in the algebra of fields, where $g_{0}^{-1} m_{0}^{2} \phi^{\mu}$ and $g_{0}{ }^{-1} m_{0}{ }^{2} \phi^{5 \mu}$ are postulated to be the physical currents. The commutation relations of $V$ and $A$ will be seen to have the correct "algebra" terms.

The expression giving $V^{0}$ and $A^{0}$ as functions of canonical variables does not contain $\gamma$, so that $V^{0}$ and $A^{0}$ automatically obey the same commutation relations as in the algebra of fields $(\gamma=0)$. Calculation of the space-time commutators is more difficult, but the

${ }^{18}$ T. D. Lee, S. Weinberg, and B. Zumino, Phys. Rev. Letters 18,1029 (1967). canonical ( $\delta$-function) part can be read off easily:

$$
\begin{aligned}
& {\left[V_{a 0}(x), V_{b i}(y)\right]} \\
& =g_{0}^{-1} m_{0}^{2}\left[V_{a 0}(x), \phi_{b i}(y)\left\{1-4 \gamma m_{0}^{2} I^{2}(y)\right\}\right] \\
& =g_{0}^{-1} m_{0}^{2}\left[V_{a 0}(x), \phi_{b i}(y)\right]\left\{1-4 \gamma m_{0}^{2} I^{2}(y)\right\} \\
& -4 \gamma g_{0}{ }^{-1} m_{0}{ }^{4} \phi_{b i}(y)\left[j_{a 0}(x)-g_{0}{ }^{-1} \partial^{j} \phi_{a 0 j}(x), I^{2}(y)\right] \\
& =i c_{a b c} V_{c i}(x) \delta(\mathbf{x}-\mathbf{y}) \\
& +i g_{0}{ }^{-2} m_{0}{ }^{2}\left\{1-4 \gamma m_{0}^{2} I^{2}(y)\right\} \partial_{x i} \delta(\mathbf{x}-\mathbf{y}) \\
& +4 \gamma g_{0}{ }^{-2} m_{0}{ }^{4} \phi_{b i}(y) \partial_{x j}\left[\phi_{a 0 j}(x), I^{2}(y)\right] \text {. } \\
& \text { Similarly, } \\
& {\left[A_{a 0}(x), A_{b i}(y)\right]} \\
& =i c_{a b c} V_{c i}(x) \delta(\mathbf{x}-\mathbf{y}) \\
& +i g_{0}{ }^{-2} m_{0}{ }^{2}\left\{1-4 \gamma m_{0}^{2} I^{2}(y)\right\} \partial_{x i} \delta(\mathbf{x}-\mathbf{y}) \\
& +4 \gamma g_{0}{ }^{-2} m_{0}{ }^{4} \phi_{b i}^{5}(y) \partial_{x j}\left[\phi^{5}{ }_{a 0 j}(x), I^{2}(y)\right], \\
& =i c_{a b c} A_{c i}(x) \delta(\mathbf{x}-\mathbf{y}) \\
& +4 \gamma g_{0}{ }^{-2} m_{0}{ }^{4} \phi^{5}{ }_{b i}(y) \partial_{x j}\left[\phi_{a 0 j}(x), I^{2}(y)\right], \\
& {\left[A_{a 0}(x), V_{b i}(y)\right]} \\
& =i c_{a b c} A_{c i}(x) \delta(\mathbf{x}-\mathbf{y}) \\
& +4 \gamma g_{0}{ }^{-2} m_{0}{ }^{4} \phi_{b i}(y) \partial_{x j}\left[\phi^{5}{ }_{a 0 j}(x), I^{2}(y)\right] .
\end{aligned}
$$

The last term in each of these commutators is a nonterminating power series in $\gamma$ and is not evaluated here, but all terms clearly have the singularity structure $\partial_{x i} \delta(\mathbf{x}-\mathbf{y})$. The time-time and space-time commutators thus have the desired $\delta$-function parts, so that $V$ and $A$ are acceptable candidates for the vector and the axial-vector currents. Note that the space-time commutators, including $\left[V_{0}, A_{i}\right]$, have ( $\gamma$-dependent) operator Schwinger terms. The space-space commutators acquire $\gamma$-dependent $\delta$-function terms as well as $\gamma$-dependent Schwinger terms.

Since the canonical commutation relations are independent of $\gamma$, one can carry out the limiting procedure of Bardakci, Frishman, and Halpern ${ }^{3}\left(g_{0}, m_{0} \rightarrow 0\right.$; $m_{0}{ }^{2} / g_{0}{ }^{2} \equiv C$ fixed) as usual, obtaining

$$
\phi_{a \mu \nu}=\phi_{a \mu \nu}^{5}=0 \text {. }
$$

Calculation of $T_{\mu \nu}$ in this limit gives

$$
T_{\mu \nu}=\left(1-4 \gamma m_{0}^{2} I^{2}\right) T^{S_{\mu \nu}}-\eta_{\mu \nu} \gamma m_{0}{ }^{4} I^{4},
$$

so that $T_{\mu \nu}$ is a simple (quartic) function of the fields. Using this form, the Schwinger condition

$$
i\left[T^{00}(x), T^{00}(y)\right]=\left[T^{0 i}(x)+T^{0 i}(y)\right] \partial_{x i} \delta(\mathbf{x}-\mathbf{y})
$$

has been explicitly checked to order $\gamma$, and can be shown to hold to all orders from general arguments. ${ }^{19}$ Solving Eq. (29) for $\phi$ and $\phi^{5}$ in terms of $V$ and $A$ then puts $T_{\mu \nu}$ in a form which is explicitly a theory of currents. We have actually carried out this procedure, but the result is not very enlightening and is not reproduced here.

${ }^{19} \mathrm{~S}$. Deser and L. K. Morrison, J. Math. Phys. (to be published). 
It is evident that this $T_{\mu \nu}$ cannot be decomposed as in Eq. (22); thus the Dashen-Frishman problem of too much symmetry is lifted, even with an arbitrarily small coupling constant $\gamma$. There is also evidence of $\boldsymbol{P}_{+} \times \boldsymbol{P}_{-}$ violation at another level: Equation (30a) and (30b) imply that, formally, $\left[V_{0}, V_{i}\right] \neq\left[A_{0}, A_{i}\right]$, so that the first Weinberg sum rule ${ }^{20}$ is not manifestly valid in this model. ${ }^{21}$

The above model, while a simple Lagrangian field theory and the simplest nonquadratic generalization of the Sugawara theory, is not so simple when viewed as a theory of currents, because the fields are complicated functions of the latter. We now describe a model that is an infinite series in the original fields, but simplifies the field-current relation. The Lagrangian is

$$
\begin{aligned}
\mathscr{L}=-\frac{1}{4}\left(\phi_{a \mu \nu} \phi_{a}{ }^{\mu \nu}+\phi^{5}{ }_{a \mu \nu} \phi^{5}{ }^{\mu \nu}\right) & \\
& +\gamma^{-1}\left[\left(1-\gamma m_{0}{ }^{2} I^{2}\right)^{-1 / 2}-1\right] .
\end{aligned}
$$

Note that an expansion for small $\gamma$ yields the Lagrangian of the algebra of fields when only the first term is kept, and the previous model (up to a rescaling of $\gamma$ ) when the first two terms are kept. The field equations are

$$
\begin{aligned}
\partial_{\nu} \phi_{a}{ }^{\mu \nu}=g_{0} c_{a b c}\left(\phi_{b}{ }^{\mu \nu} \phi_{c \nu}+\right. & \left.\phi^{5} b^{\mu \nu} \phi^{5}{ }_{c \nu}\right) \\
& -m_{0}{ }^{2} \phi_{a}{ }^{\mu}\left[1-\gamma m_{0}{ }^{2} I^{2}\right]^{-1 / 2}, \\
\partial_{\nu} \phi^{5}{ }_{a}{ }^{\mu \nu}=g_{0} c_{a b c}\left(\phi_{b}{ }^{\mu \nu} \phi^{5}{ }_{c \nu}+\right. & \left.\phi^{5}{ }_{b}{ }^{\mu \nu} \phi_{c \nu}\right) \\
& -m_{0}{ }^{2} \phi^{5}{ }_{a}^{\mu}\left[1-\gamma m_{0}{ }^{2} I^{2}\right]^{-1 / 2} .
\end{aligned}
$$

One concludes as before that the vector and the axialvector currents, defined by

$$
\begin{aligned}
& V_{a^{\mu}}=g_{0}^{-1} m_{0}{ }^{2} \phi_{a}{ }^{\mu}\left(1-\gamma m_{0}{ }^{2} I^{2}\right)^{-1 / 2}, \\
& A_{a^{\mu}}=g_{0}{ }^{-1} m_{0}{ }^{2} \phi^{5} a^{\mu}\left(1-\gamma m_{0}{ }^{2} I^{2}\right)^{-1 / 2},
\end{aligned}
$$

are conserved. These can be easily inverted to yield

$$
\begin{aligned}
\phi_{a}{ }^{\mu}= & g_{0} m_{0}{ }^{-2} V_{a^{\mu}} \\
& \times\left[1+\gamma g_{0}{ }^{2} m_{0}{ }^{-2}\left(V_{a \nu} V_{a^{\nu}}+A_{a \nu} A_{a}{ }^{\nu}\right)\right]^{-1 / 2}, \\
\phi^{5} a^{\mu}=g_{0} m_{0}{ }^{-2} A_{a}{ }^{\mu} & \\
\times & \left.\times 1+\gamma g_{0}{ }^{2} m_{0}{ }^{-2}\left(V_{a \nu} V_{a}{ }^{\nu}+A_{a \nu} A_{a^{\nu}}\right)\right]^{-1 / 2} .
\end{aligned}
$$

When the limit $g_{0}, m_{0} \rightarrow 0\left(m_{0}^{2} / g_{0}^{2}=C\right.$ fixed $)$ is taken, the resulting $T_{\mu \nu}$ can be written as a simple function of $V_{a \mu}$ and $A_{a \mu}$. The result is

$$
\begin{aligned}
& T_{\mu \nu}= C^{-1}\left(V_{a \mu} V_{a \nu}+A_{a \mu} A_{a \nu}\right) \\
& \times\left[1+\gamma C^{-1}\left(V_{a \mu} V_{a^{\mu}}+A_{a \mu} A_{a}{ }^{\mu}\right)\right]^{-1 / 2} \\
&+\eta_{\mu \nu} \gamma^{-1}\left\{\left[1+\gamma C^{-1}\left(V_{a \mu} V_{a}{ }^{\mu}+A_{a \mu} A_{a}{ }^{\mu}\right)\right]^{-1 / 2}-1\right\} .
\end{aligned}
$$

An analysis, identical to the one for the quartic model, shows that the algebra ( $\delta$-function) part of the timetime and space-time commutators of the currents are

\footnotetext{
${ }^{20}$ S. Weinberg, Phys. Rev. Letters 18, 507 (1967).

21 The equality of the vector and the axial-vector two-point functions is another formal problem of Sugawara's theory: If one insists on strict $S U_{2} \times S U_{2}$ invariance, the theory cannot accommodate both the existence of Goldstone pions (Goldstone because $\partial_{\mu} A^{\mu}=0$ ) and the identification of $V_{3}$ with the isovector part of the electromagnetic current; see S. Deser and J. Rawls, Phys. Letters 27B, 460 (1968).
}

as desired. In fact, the conclusions following Eq. (30) also apply to this theory, as does the conclusion that the $\mathcal{P}_{+} \times \mathcal{P}_{-}$degeneracy is lifted.

\section{APPENDIX}

We generalize the $\phi \bar{F}^{\mu \nu} F_{\mu \nu}$ coupling of Sec. II to $S U_{2} \times S U_{2}$. In the $S U_{2} \times S U_{2}$ Sugawara theory,

$$
\begin{aligned}
& T_{\mu \nu}=C^{-1}\left[\left(V^{a}{ }_{\mu} V^{a}{ }_{\nu}+A^{a}{ }_{\mu} A^{a}{ }_{\nu}\right)\right. \\
& \left.-\frac{1}{2} \eta_{\mu \nu}\left(V^{a}{ }_{\lambda} V^{a \lambda}+A^{a}{ }_{\lambda} A^{a \lambda}\right)\right] \\
& \mathcal{L}=-\frac{1}{2} C^{-1}\left(V^{a}{ }_{\mu} V^{a \mu}+A^{a}{ }_{\mu} A^{a \mu}\right),
\end{aligned}
$$

with the field equations

$$
\begin{gathered}
\partial^{\mu} V^{a}{ }_{\mu}=0, \quad \partial^{\mu} A^{a}{ }_{\mu}=0, \\
\partial_{\mu} V^{a_{\nu}}-\partial_{\nu} V^{a}{ }_{\mu}=\frac{1}{2} C^{-1} \epsilon^{a b c}\left(V^{b}{ }_{\mu} V^{c}{ }_{\nu}+A^{b}{ }_{\mu} A^{c}{ }_{\nu}\right), \\
\partial_{\mu} A^{a}{ }_{\nu}-\partial_{\nu} A^{a}{ }_{\mu}=\frac{1}{2} C^{-1} \epsilon^{a b c}\left(V^{b}{ }_{\mu} A^{c}{ }_{\nu}+A^{b}{ }_{\mu} V^{c}{ }_{\nu}\right) .
\end{gathered}
$$

In terms of ${ }^{12}$

$$
J^{a}{ }_{ \pm \mu} \equiv \frac{1}{2}\left(V^{a}{ }_{\mu} \pm A^{a}{ }_{\mu}\right),
$$

Eqs. (A1-A4) become

where

$$
\begin{aligned}
& T_{\mu \nu}=T^{+}{ }_{\mu \nu}+T^{-}{ }_{\mu \nu}, \\
& T^{ \pm}{ }_{\mu \nu}=2 C^{-1}\left(J^{a}{ }_{ \pm \mu} J^{a}{ }_{ \pm \nu}-\frac{1}{2} \eta_{\mu \nu} J^{a}{ }_{ \pm \lambda} J^{a}{ }_{ \pm}^{\lambda}\right) \text {, } \\
& \mathfrak{L}=-C^{-1}\left(J^{a}{ }_{+\mu} J^{a}+{ }^{\mu}+J^{a}{ }_{-\mu} J^{a}{ }_{-}{ }^{\mu}\right), \\
& \partial^{\mu} J_{ \pm \mu}^{a}=0, \\
& \partial_{\mu} J_{ \pm \nu}^{a}-\partial_{\nu} J_{ \pm \mu}^{a}=2 C^{-1} \epsilon^{a b c} J^{b}{ }_{ \pm \mu} J_{ \pm \nu}^{c} .
\end{aligned}
$$

Thus, $J_{+}$and $J_{-}$give rise to independent $S U_{2}$ Sugawara models, and may be separately represented in terms of independent scalar isovectors $S^{\mathrm{a}}{ }_{+}$and $S^{\mathrm{a}}-$; one $e^{4,11}$ representation is

$$
\begin{gathered}
J^{a}{ }_{ \pm \mu}=C\left\{\epsilon^{a b c} S^{b}{ }_{ \pm} \partial_{\mu} S^{c}{ }_{ \pm}+S^{a}{ }_{ \pm} \partial_{\mu} S^{0}{ }_{ \pm}-S^{0}{ }_{ \pm} \partial_{\mu} S^{a}{ }_{ \pm}\right\}, \\
S^{0}{ }_{ \pm} \equiv\left(1-S_{ \pm}{ }^{2}{ }^{1 / 2}, \quad S_{ \pm}{ }^{2} \equiv S^{a}{ }_{ \pm} S^{a}{ }_{ \pm},\right.
\end{gathered}
$$

where $S^{\text {a }} \pm$ transform under parity according to $P S^{\mathrm{a}, 0}{ }_{ \pm} P^{-1}=S^{\mathrm{a}, 0} \mp$. We now add to the Lagrangian of Eq. (A8) the interaction

$\mathfrak{L}_{\mathrm{int}}=-(\alpha / 4 \pi) C^{-1} \tilde{F}^{\mu \nu} F_{\mu \nu}$

$\times\left(\arcsin \frac{S_{+}^{3}}{\left[1-\left(S_{+}^{1}\right)^{2}\right]^{1 / 2}}-\arcsin \frac{S^{3}-}{\left[1-\left(S_{-}^{1}\right)^{2}\right]^{1 / 2}}\right)$,

where

$$
\left(S^{1}\right)^{2}=S_{ \pm}^{1} S_{ \pm}^{1}+S_{ \pm}^{2} S^{2}{ }^{2}
$$

As in the Abelian case, this term does not contribute to $T_{\mu \nu}$ Equation (A4) is unchanged, and the neutral components of Eq. (A3) are now of the desired form

$$
\partial^{\mu} V^{3}{ }_{\mu}=0, \quad \partial^{\mu} A^{3}{ }_{\mu}=(\alpha / 2 \pi) \bar{F}^{\mu \nu} F_{\mu \nu} .
$$

However, the divergences of the charged currents no longer vanish either and are quite complicated. The new terms are of order $\alpha$, as against the order $\alpha^{1 / 2}$ minimal coupling contributions to these divergences. 